\title{
Soliton excitations in Josephson tunnel junctions
}

\author{
Lomdahl, P. S.; Sørensen, O. H.; Christiansen, Peter Leth
}

Published in:

Physical Review B

Link to article, DOI:

10.1103/PhysRevB.25.5737

Publication date:

1982

Document Version

Publisher's PDF, also known as Version of record

Link back to DTU Orbit

Citation (APA):

Lomdahl, P. S., Sørensen, O. H., \& Christiansen, P. L. (1982). Soliton excitations in Josephson tunnel junctions. Physical Review B, 25(9), 5737-5748. https://doi.org/10.1103/PhysRevB.25.5737

\section{General rights}

Copyright and moral rights for the publications made accessible in the public portal are retained by the authors and/or other copyright owners and it is a condition of accessing publications that users recognise and abide by the legal requirements associated with these rights.

- Users may download and print one copy of any publication from the public portal for the purpose of private study or research.

- You may not further distribute the material or use it for any profit-making activity or commercial gain

- You may freely distribute the URL identifying the publication in the public portal

If you believe that this document breaches copyright please contact us providing details, and we will remove access to the work immediately and investigate your claim. 


\title{
Soliton excitations in Josephson tunnel junctions
}

\author{
P. S. Lomdahl,* O. H. Soerensen, ${ }^{\dagger}$ and P. L. Christiansen \\ Laboratory of Applied Mathematical Physics, The Technical University of Denmark, \\ DK-2800 Lyngby, Denmark
}

(Received 17 November 1981)

\begin{abstract}
A detailed numerical study of a sine-Gordon model of the Josephson tunnel junction is compared with experimental measurements on junctions with different $L / \lambda_{J}$ ratios. The soliton picture is found to apply well on both relatively long $\left(L / \lambda_{J}=6\right)$ and intermediate $\left(L / \lambda_{J}=2\right)$ junctions. We find good agreement for the current-voltage characteristics, power output, and for the shape and height of the zero-field steps (ZFS). Two distinct modes of soliton oscillations are observed: (i) a bunched or congealed mode giving rise to the fundamental frequency $f_{1}$ on all ZFS's and (ii) a "symmetric" mode which on the $N$ th ZFS yields the frequency $N f_{1}$. Coexistence of two adjacent frequencies is found on the third ZFS of the longer junction $\left(L / \lambda_{J}=6\right)$ in a narrow range of bias current as also found in the experiments. Small asymmetries in the experimental environment, a weak magnetic field, e.g., is introduced via the boundary conditions of our numerical model. This gives a junction response to variations in the applied bias current close to that observed experimentally.
\end{abstract}

\section{INTRODUCTION}

The study of soliton dynamics in connection with large Josephson tunnel junctions has recently drawn considerable theoretical ${ }^{1-6}$ and experimen$\operatorname{tal}^{7,8}$ attention. Fulton and Dynes ${ }^{1}$ conceived the idea that the Josephson tunnel junction could support the resonant propagation of a soliton (or fluxon) trapped in the junction. The soliton being a $2 \pi$ jump in the phase difference $(\phi)$ across the insulating barrier which separates the two superconductors. The moving soliton is accompanied by a voltage pulse $\left(\sim \phi_{t}\right)$ which can be detected at either end of the junction. The dc manifestation of the motion is a sequence of equidistantly spaced branches in the current-voltage characteristic of the junction. These near-constant voltage branches which were first reported by Chen, Finnegan, and Langenberg ${ }^{9}$ are known as zero-field steps (ZFS) because they occur in the absence of an external magnetic field. In contrast, the so-called Fiske steps are found only when a magnetic field is applied. ${ }^{10}$ The ZFS's appear at voltages given by $V=N \Phi_{0} \bar{c} / L$, where $N$ is an integer, the flux quantum $\Phi_{0}=h / 2 e=2.064 \times 10^{-15} \mathrm{~Wb}, \bar{c}$ is the electromagnetic wave velocity in the junction, and $L$ is the length of the junction. On the first ZFS $(N=1)$ a single soliton pendles back and forth along the junction with a velocity $\cong \bar{c}$, hence producing a periodic voltage-pulse train of frequency $f_{1}=\bar{c} / 2 L$ at both ends. On the $N$ th ZFS the motion of $N$ solitons is involved and $N$ pulses are produced within one period $1 / f_{1}$. The detailed frequency spectrum of the voltage will depend on the distribution in time of the $N$ pulses within the period. Analog ${ }^{4,6}$ and numerical studies ${ }^{5}$ have suggested that configurations where the solitons are bunched (or congealed) play an important role in explaining the dynamics of the motion. This is supported by recent detailed measurements ${ }^{7}$ on niobium-lead junctions and by comparison with numerical siumulation ${ }^{11}$ with parameters chosen in accordance with experiments.

In this paper we report on the detailed numerical study and comparison with the experiments on two junctions with different length-to-Josephsonpenetration-depth $\left(L / \lambda_{J}\right)$ ratios. We demonstrate that the shunted-junction (SJ) model, which leads to a perturbed sine-Gordon equation, ${ }^{14}$ explains the experimental observations quantitatively even down to very subtle details. We find good agreement for the current-voltage characteristic, power output, and for the shape and height of the ZFS's. On the second ZFS we find two distinct modes of soliton oscillations, the perfectly symmetric mode and the bunched mode, thus confirming previous results by 
Erné and Parmentier. On the third ZFS, the highest step considered here, two simultaneous frequencies are observed. This was also seen in the experiments. By introducing a weak external field in the numerical model we are able to reproduce the manner in which the junction responds to changes in the applied bias current.

The outline of the paper is as follows. In Sec. II we present the SJ model, derive the perturbed sine-Gordon equation, and give the parameters relevant to the experimental data. In Sec. III we present the results for the longer junction $\left(L / \lambda_{J}=6\right)$ current biased on the first, second, and third ZFS's, respectively. In Sec. IV similar results are described for the shorter junction $\left(L / \lambda_{J}=2\right)$. Finally in Sec. V we give our conclusion. Our numerical method is discussed in the Appendix.

\section{THE PERTURBED SINE-GORDON EQUATION AND THE SJ MODEL}

An overlap-geometry Josephson tunnel junction consists of two superconductivity metal layers separated by a thin insulating oxide layer of uniform thickness $\left(t_{\mathrm{ox}}\right)$ that is small enough to permit quantum-mechanical tunneling of electrons. The geometry is shown in Fig. 1(a). We shall restrict our attention to narrow junctions with width $\left(W<<\lambda_{J}\right)$ which permits us to treat the elec-

(a)

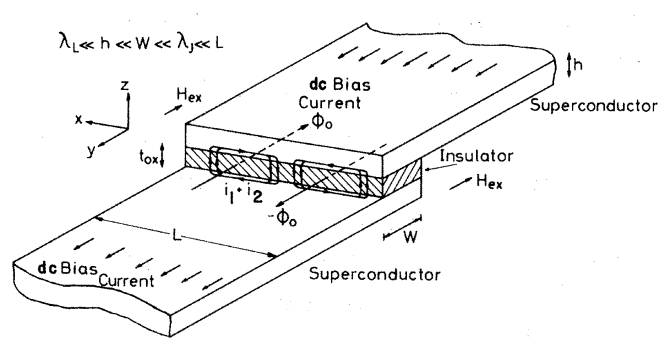

(b)

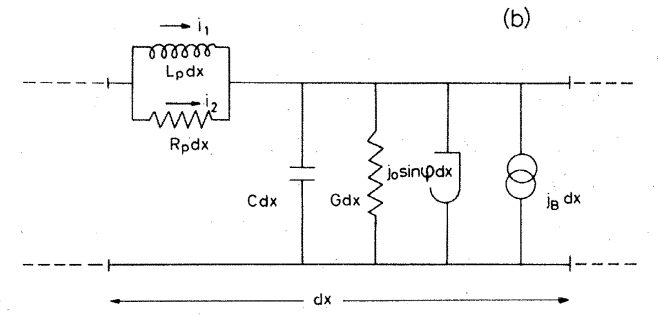

FIG. 1. (a) Josephson tunnel junction of overlap geometry. (Not drawn to scale.) (b) Element of lumped transmission-line equivalent circuit representing the $\mathbf{S J}$ model. tromagnetic fields in the insulating barrier as uniform in the $Y$ coordinate. ${ }^{12}$ In particular, this means that the applied bias current can be considered uniformly distributed along the entire $X$ axis. The orientation of the coordinate system is also shown in Fig. 1(a). The tunneling supercurrent is described by the two basic Josephson equations, ${ }^{13}$

$$
\begin{aligned}
& j(X)=j_{0} \sin \phi, \\
& \frac{\partial \phi}{\partial T}=\frac{2 e}{\hbar} V=\frac{2 \pi}{\Phi_{0}} V,
\end{aligned}
$$

where $T$ is laboratory time.

Here, $j(X)$ is the Josephson current crossing the barrier per unit length in the $X$ direction, $j_{0}$ being the maximum value. Formally, $\phi=\phi(X, T)$ is the difference between the phases of the order parameters of the two superconductors. It is however, more convenient for our purpose to define it through the second of the Josephson equations, Eq. (2.1b). Here, $V=V(X, T)$ is the voltage drop across the insulating barrier. A combination of Eqs. (2.1a) and (2.1b) with the Maxwell equations yields the nonlinear set of partial-differential equations representing the dynamics of the junction ${ }^{14,15}$ [cf. Fig. 1(a)],

$$
\begin{aligned}
\frac{\partial V}{\partial X}=-i_{2} R_{P} & =-L_{P} \frac{\partial i_{1}}{\partial T} \\
\frac{\partial i_{1}}{\partial X}+\frac{\partial i_{2}}{\partial X}= & -C \frac{\partial V}{\partial T}-G V \\
& -j_{0} \sin \phi+j_{B}
\end{aligned}
$$

Equations (2.1b), (2.2a), and (2.2b) also model the lumped transmission-line equivalent circuit in Fig. 1 (b). It is this model which is often referred to as the SJ model. In Eqs. (2.2a) and (2.2b) $i_{1}$ and $i_{2}$ are the superconducting and the normal components of the current flowing parallel to the barrier in the $X$ direction. The inductance per unit length $L_{P}$ represents the magnetic energy storted within one London penetration depth of the superconducting metal films. It is given by ${ }^{16}$

$$
L_{P}=\mu_{0}\left(2 \lambda_{L}+t_{\mathrm{ox}}\right) / W,
$$

where $\lambda_{L}$ is the average London distance for the two superconductors (here niobium and lead), and $\mu_{0}=4 \pi \times 10^{-7} \mathrm{H} / \mathrm{m}$. The series resistance per unit length $R_{P}$ represents scattering of quariparticles in the surface layers of the two superconductors. The capacitance per unit length $C$ represents the elec- 
tric energy stored in the barrier and is given by

$$
C=\epsilon_{r} \epsilon_{0} W / t_{\mathrm{ox}},
$$

where $\epsilon_{r}$ is the relative dielectric constant of the barrier oxide layer and $\epsilon_{0}=1 / 36 \pi \times 10^{-9} \mathrm{~F} / \mathrm{m}$.

The dissipative currents per unit length (the quasiparticle tunneling) are represented by the term $G V$, where $1 / G$ is an effective normal resistance. The supercurrent per unit length is given by $j_{0} \sin \phi$. Finally $j_{B}$ represents an externally applied bias current per unit length.

Equations (2.1b), (2.2a), and (2.2b) combine into the third-order partial-differential equation

$$
\begin{gathered}
\left(L_{P} / R_{P}\right) \phi_{X X T}+\phi_{X X}-L_{P} C \phi_{T T}-G L_{P} \phi_{T} \\
=\left(2 \pi L_{P} j_{0} / \Phi_{0}\right)\left(\sin \phi-j_{B} / j_{0}\right) .
\end{gathered}
$$

Introduction of normalized space and time coordinates, $x$ and $t$, related to laboratory coordinates by

$$
\begin{aligned}
& x=X / \lambda_{J}, \\
& t=T \omega_{0},
\end{aligned}
$$

where $\lambda_{J}$ is the Josephson length,

$$
\lambda_{J}=\left(\Phi_{0} / 2 \pi j_{0} L_{P}\right)^{1 / 2},
$$

and $\omega_{0}$ is the Josephson plasma frequency

$$
\omega_{0}=\left(2 \pi j_{0} / \Phi_{0} C\right)^{1 / 2},
$$

yields

$$
\beta \phi_{x x t}+\phi_{x x}-\phi_{t t}-\alpha \phi_{t}=\sin \phi-\gamma .
$$

The constants $\alpha, \beta$, and $\gamma$ are defined by

$$
\begin{aligned}
& \alpha=\frac{G}{\omega_{0} C}, \\
& \beta=\frac{\omega_{0} L_{P}}{R_{p}}, \\
& \gamma=\frac{j_{B}}{j_{0}} .
\end{aligned}
$$

The values of the two parameters $\lambda_{J}$ and $\omega_{0}$ are especially important for the dynamics of the junction. The Josephson plasma frequency $\omega_{0}$ is the lowest possible frequency of small-amplitude oscillations in the unbiased junction, whereas the Josephson length $\lambda_{J}$ can be viewed as a screening length over which the magnetic field induced by the Josephson supercurrent causes noticeable changes in $\phi .{ }^{15}$ The propagation velocity of electromagnetic signals in the junction is $\bar{c}=\lambda_{J} \omega_{0}$ $=\left(L_{P} C\right)^{-1 / 2}$. The space-time behavior of the voltages and currents is governed by Eq. (2.10), which without the dissipative terms and the external bias current is the famed sine-Gordon equation with well-known soliton solutions. This fact will be used in the numerical computations presented in Sec. III.

Using the normalized time $t$, we rewrite Eq. (2.1b) as

$$
V=\left(\Phi_{0} \omega_{0} / 2 \pi\right) \phi_{t} \equiv V_{N} \phi_{t} .
$$

Further we mention that Eqs. (2.6) and (2.8), combined with the time integral of Eq. (2.2a), yields

$$
i_{1}=-\left(j_{0} \lambda_{J}\right) \phi_{x} \equiv-I_{N} \phi_{x} .
$$

Equation (2.15) can be used to establish the boundary conditions for the junction. The superconducting surface current $i_{1}$ gives rise to a magnetic field perpendicular to the length direction of the junction. Thus, fixing an external magnetic field in the $Y$ direction $\left(H_{\mathrm{ex}}\right)$ at the two ends of the junction corresponds to specifying $\phi_{x}$ at these points. Considering a junction of length $l=L / \lambda_{J}$, we get

$$
\phi_{x}(0, t)=\phi_{x}(l, t)=-\frac{H_{\mathrm{ex}} W}{I_{N}} \equiv \eta .
$$

Here, the dimensionless quantity $\eta$ represents the external field. In most of our computations we have assumed "open-circuit" boundary conditions, i.e., $\eta=0$, thus neglecting loading effects due to radiation loss. However, in order to model asymmetries in the experimental system due to small external magnetic fields, e.g., we have sometimes used values of $\eta$ different from zero.

The quasiparticle tunneling-loss parameter $\alpha$ is determined through Eq. (2.11) from the slope $(G)$ of the linear part of the experimental $I-V$ curve. Owing to the nonlinear nature of this curve, $\alpha$ cannot be determined unambiguously. We assume $\alpha$ to be constant in the range $0.01<\alpha<0.05$ for the junctions considered here. The parameter $\beta$ is determined by Eq. (2.12) which is also the inverse of the $Q$ of the superconductor surface impedance at $\omega_{0}$ and describes the quasiparticle losses in the film electrodes. The value of $\beta$ is readily obtainable from recent measurements. ${ }^{17}$ Relevant junction parameters are listed in Table I.

\section{RESULTS FOR THE LONGER JUNCTION $(l=6)$}

The experimentally determined parameters in Table I have been used in our numerical solution 
TABLE I. Junction parameters used in the calculations.

\begin{tabular}{lll}
\hline \hline Sample & \multicolumn{1}{c}{1} & \multicolumn{1}{c}{2} \\
\hline$L$ & $973 \mu \mathrm{m}$ & $467 \mu \mathrm{m}$ \\
$W$ & $119 \mu \mathrm{m}$ & $67 \mu \mathrm{m}$ \\
$l$ & $\sim 6$ & 2 \\
$\alpha$ & 0.05 & 0.012 \\
$\beta$ & 0.02 & 0.01 \\
$\lambda_{J}$ & $1.56 \times 10^{-4} \mathrm{~m}$ & $2.25 \times 10^{-4} \mathrm{~m}$ \\
$\omega_{0}$ & $5.8 \times 10^{10} \mathrm{~s}^{-1}$ & $4.0 \times 10^{10} \mathrm{~s}^{-1}$ \\
\hline \hline
\end{tabular}

of Eq. (2.10). From a Fourier analysis of $\phi_{t}(l, t)$ we obtain the dc $I-V$ characteristic and the harmonic contents of the voltage excited at the end of the junction. These numerical results are then compared with the corresponding experimental results. From Eq. (2.14) follows that the zero-order Fourier component (average value) of $\phi_{t}$ corresponds to the junction dc voltage. Thus, a plot of $\left\langle\phi_{t}\right\rangle V_{N}$ versus the applied bias current $I_{\mathrm{dc}}=j_{B} L$ will correspond to the experimental $I-V$ characteristic of the junction. The higher-order Fourier components of $\phi_{t}(l, t)$ will give us information on the frequencies excited.

Before we go into the detailed discussion of our results we draw attention to the following basic experimental observation: On all the observed ZFS's in the $I-V$ characteristic, radiation with the same fundamental frequency $f_{1}=\bar{c} / 2 L$ is found. This is somewhat unexpected, because $a$ priori one might expect that the $N$ soliton mode on the $N$ th ZFS would have the solitons arrive at either end of the junction evently distributed in time, thus leading to a fundamental frequency of $N f_{1}$. The experimental finding of the frequency $f_{1}$ on all the ZFS's can be accounted for by introducing the notion of congealed or bunched solitons. ${ }^{7}$ Instead of $N$ individual solitons, one or several bunches of solitons are formed, giving rise to an excitation with the fundamental frequency $f_{1}$. Such bunches of solitons are indeed what we observe in the numerical solutions of Eq. (2.10). The dc manifestation of our computations is shown in Fig. 2 for the first three ZFS's. The experimental result ${ }^{7}$ is shown as a full curve. Higher-order steps $(N>3)$ have also been found experimentally, but we have not pursued these numerically. Figure 2 shows good agreement between numerical and experimental results, in regard to height, shape, and position of

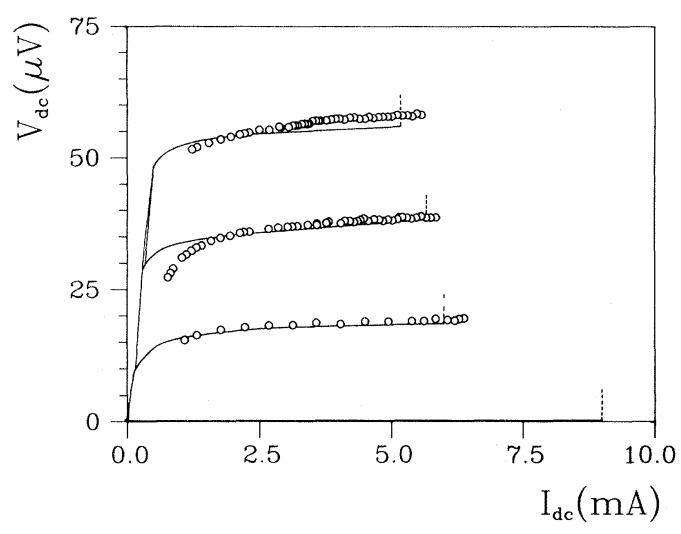

FIG. 2. dc voltage vs applied bias current for sample $1(l=6)$ showing the first three ZFS's. Circles indicate the numerical results and solid lines represent the experimental results.

the steps. We shall now discuss the dynamics on the individual steps.

\section{A. First ZFS}

In Fig. 3 we show a typical numerical result of the oscillatory motion of one soliton after steady state has been reached. [Defined operationally as the state which occurs when the first four Fourier components of $\phi_{t}(l, t)$ attained a constant value to within $2 \%$.] For runs on the first ZFS this state in general occurred after 1200 normalized time units. As initial conditions we have used the onesoliton solution of the unperturbed sine-Gordon equation augmented by the "ground-state" solution $\phi(x, t)=\sin ^{-1} \gamma$, in order to prevent unnecessary

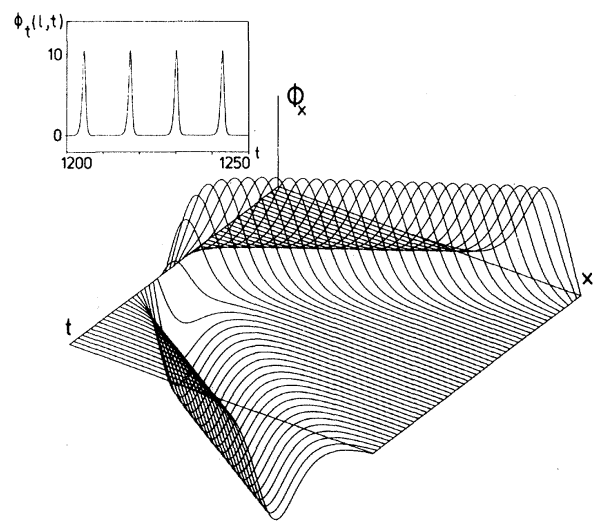

FIG. 3. Numerical solution of Eq. (2.10) with $\gamma=0.35, \alpha=0.05, \beta=0.02, \eta=0$, and $l=6$. Approximately one period of oscillation on the first ZFS is plotted in terms of $\phi_{x}(x, t)$ for 10 time units. The inset shows $\phi_{t}(l, t)$ for 50 time units. 
generation of plasmons. ${ }^{18}$ In order to obtain soliton confinement on the junction the soliton was launched with initial velocity equal to 0.9 . The inset of Fig. 3 shows the voltage pulse train at $x=l$ after repeated reflections.

In Fig. 4 we show the harmonic contents of the power versus the applied dc bias current. The power is here defined as the square of the Fourier components of the voltage in arbitrary units. The oscillation is found to be stable for $0.1<\gamma<0.71$. For $\gamma>0.71$ the soliton disappears and the solution switches to a spatially uniform $\phi$ excitation. (In the $I-V$ curve this change corresponds to a jump from the ZFS to the Ohmic background.) For $\gamma<0.1$ a transition to a static solution is found. (In the $I-V$ curve this change corresponds to a jump from the ZFS to a point on the axis $\left\langle\phi_{t}\right\rangle=0$ ). From Fig. 4 it is clear that the ratio of the first three harmonics is nearly constant over a wide range of bias values. However, for the higher values just below switching, the contents of all the harmonics is nearly equal. This is of course a manifestation of the fact that the soliton shape approaches the step function when velocity tends to unity. We have not observed the irregular behavior for small $\gamma$ values found by Erné and Parmentier [Ref. 5, second listing, Fig. 5(a)].

\section{B. Second ZFS}

When two solitons are involved in the oscillations on the junction the picture becomes more complex. Two distinct ways of operation are now possible, the symmetric mode and the bunched mode shown in Figs. 5 and 6, respectively. From the insets it is clear that the frequency doubles when we pass from the bunched mode to the symmetric mode. In Fig. 7 the harmonic contents of

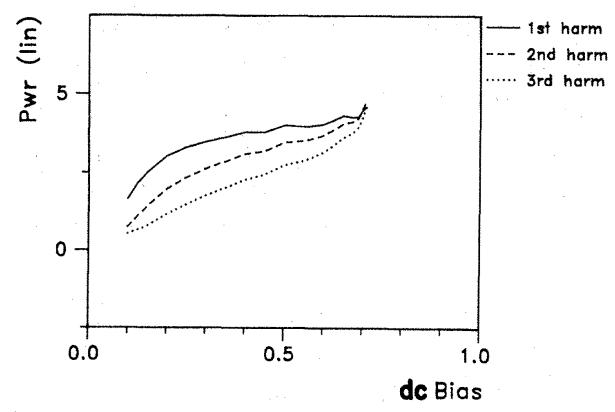

FIG. 4. Harmonic contents of power vs the dc bias $\gamma$ on the first ZFS. Obtained by numerical solution of Eq. (2.10) with parameters for sample 1.

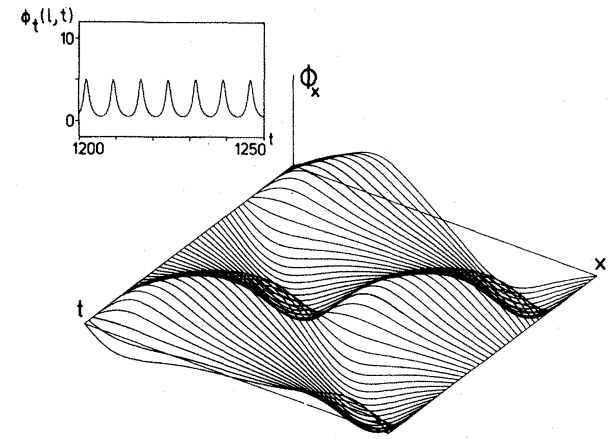

FIG. 5. Numerical solution of Eq. (2.10) with $\gamma=0.125$, $\alpha=0.05, \beta=0.02, \eta=0$, and $l=6$, showing the symmetric mode on the second ZFS in terms of $\phi_{x}(x, t)$ for 10 time units. The inset shows $\phi_{t}(l, t)$ for 50 time units.

the power is shown as function of the dc bias current. The figure was obtained in the following way: For $\gamma=0.5$ (Ref. 19), we use the initial conditions consisting of two superimposed sineGordon one-soliton solutions (plus the $\sin ^{-1} \gamma$ term) launched with equal velocity 0.9 . This choice of initial conditions corresponds to a bunched mode. When steady state was obtained for $(t \cong 2000)$ the computations were stopped, the bias current was increased by 0.01 (i.e., to $\gamma=0.51$ ), and the computation restarted, the initial conditions now being the steady-state solution obtained for $\gamma=0.5$. This procedure was continued until a switched from the second ZFS to the background curve occurred for $\gamma=0.65$. This is the upper limit in $\gamma$ for stability of the bunched mode. The process was now reversed and the bias current was decreased in steps (0.01). Continuing downwards we found transition

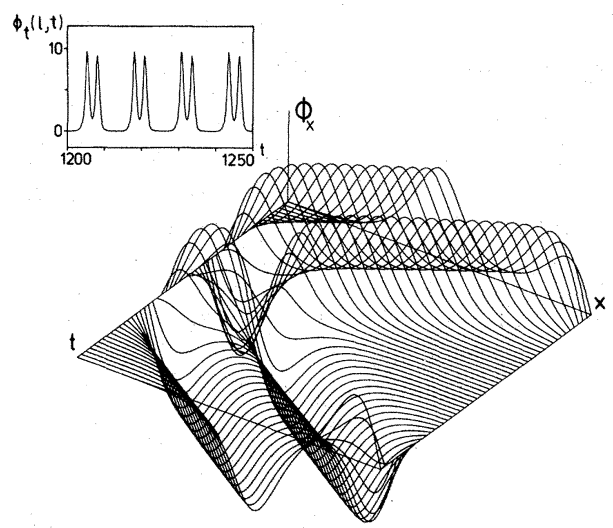

FIG. 6. Numerical solution of Eq. (2.10) with $\gamma=0.3$, $\alpha=0.05, \beta=0.02, \eta=0$, and $l=6$, showing the bunched mode on the second ZFS in terms of $\phi_{x}(x, t)$ for 10 time units. The inset shows $\phi_{t}(l, t)$ for 50 time units. 


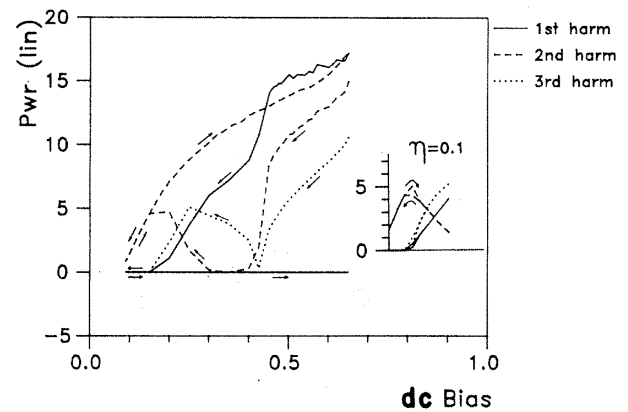

FIG. 7. Harmonic contents of power vs the dc bias $\gamma$ on the second ZFS. Obtained by numerical solution of Eq. (2.10) with parameters for sample 1. The arrows indicate the direction in which the bias current was varied. The inset shows the near vanishing of hysteresis when a small external magnetic field is applied $(\eta=0.1)$.

to the symmetric mode at $\gamma=0.16$. In Fig. 7 the first and third harmonics vanish for this value of $\gamma$. At $\gamma=0.09$ the lower limit of stability was reached and the oscillations extinguished. When $\gamma$ was increased again, the symmetric mode remained stable even beyond the point where the transition from bunched to symmetric mode occurred for decreasing bias. The symmetric mode finally switched from the second ZFS into the solution at the background curve at the same point as the bunched mode did $(\gamma=0.65)$. Thus, we have found that over a large range of $\gamma$ values both the symmetric and the bunched mode are stable. Further computations for $\gamma=0.25$ confirmed the stability of the symmetric mode as well as the bunched mode, with symmetric and bunched solitons in the initial conditions, respectively. In Figs. 8(a) and 8(b) the harmonic in the power are shown as a function of time in the two cases. After a transient the harmonics settle down at constant values. In Fig. 8(a) the second harmonic is dominant, corresponding to the symmetric mode. The ripples on the first and third harmonics $(50 \mathrm{~dB}$ below) are due to numerical noise. In Fig. 8(b) the bunched mode is characterized by the dominant first and third harmonics.

We emphasis that our computational way of changing $\gamma$ simulates the experimental way of regulating the bias current. ${ }^{7}$ Thus, the $I-V$ characteristic (Fig. 2) has been obtained experimentally be careful sweeps in the bias current. The change from a bunched mode into a symmtric mode for decreasing values of $\gamma$ has also been found experimentally. However, starting in a symmetric mode and increasing $\gamma$ we observe no spontaneous transition to the bunched mode as is found experimen-
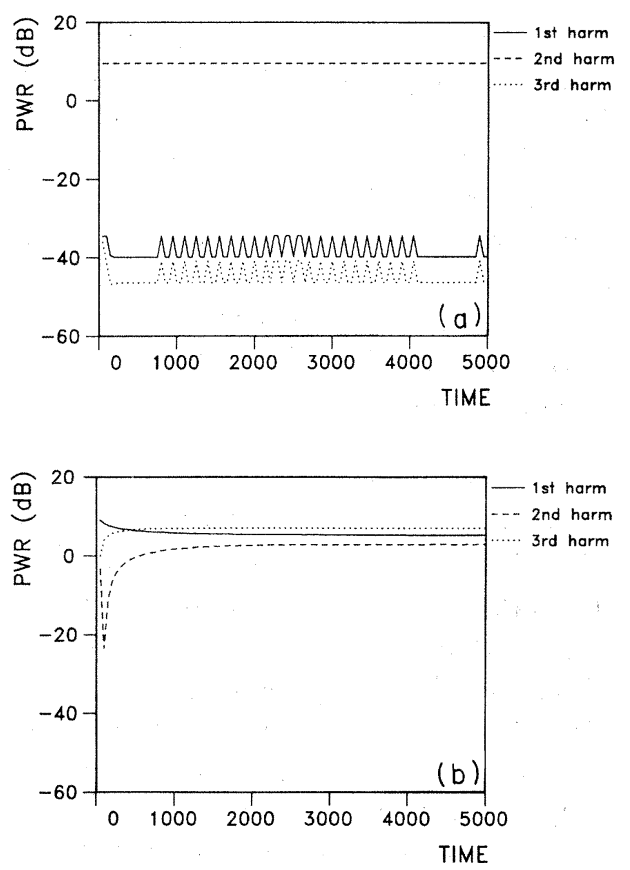

FIG. 8. Harmonics in power vs time on the second ZFS obtained by numerical solution of Eq. (2.10) with $\gamma=0.25, \alpha=0.05, \beta=0.02, \eta=0$, and $l=6$. (a) depicts initial conditions representing a symmetric mode. (b) shows initial conditions representing a bunched mode. Both modes are stable.

tally. In the experimental setup small asymmetries due to weak external magnetic fields cannot be completely ruled out. In our calculations we model this feature by introducing nonzero boundary conditions [e.g., $\eta=0.1$ in Eq. (2.17)]. ${ }^{20}$ In the inset in Fig. 7 the resulting transition region between bunched and symmetric modes is shown. Only a small reminiscent of the hysteresis phenomenon observed for $\eta=0$ is left and a reversible transition from symmetric to bunched mode is found for $\gamma=0.16$. The spontaneous transition is also shown in Fig. 9, where the effect of a gradual increase of the external magnetic field is illustrated for a fixed value of bias current $(\gamma=0.25)$. The figure was obtained in the following way: At $t=-2500$ we started a symmetric mode, i.e., two sine-Gordon solitons launched with velocities 0.9 and -0.9 , respectively. For $-2500<t<-1250$ (not shown) we kept $\eta=0$. At $t=-1250$ the magnetic field $\eta$ was increased to 0.05 . The computations were then continued until $t=0$. At $t=0$ the magnetic field was raised to $\eta=0.1$, and the results up to $t=1000$ are shown in the figure. For $0<t<5000$ we see a rapid increase towards a con- 


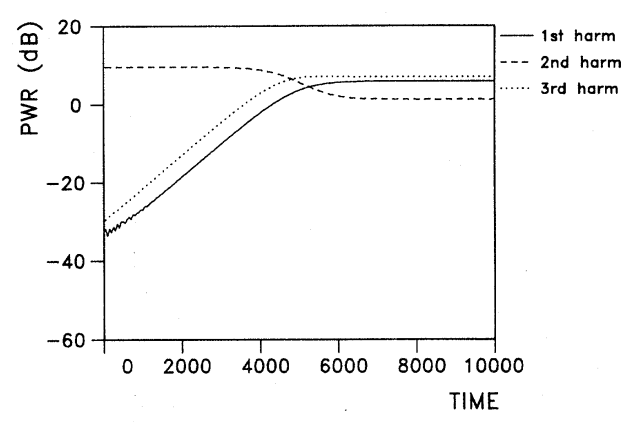

FIG. 9. Transition from the symmetric to the bunched mode when an external magnetic field is gradually increased up to $\eta=0.1$ (see text). The other parameters are as in Fig. 8.

stant value in the first and third harmonics. In the interval $4000<t<6000$ the second harmonic drops $10 \mathrm{~dB}$. These changes signify a transition from symmetric to bunched mode at $t \cong 5000$. The final levels of the harmonics compare with levels in Fig. 8(b).

\section{Third ZFS}

Most phenomena observed on the second ZFS reappear on the third ZFS where three solitons are simultaneously excited on the junction. In Figs. 10 and 11 the symmetric mode (three single solitons) and the bunched mode (three bunched solitons) are shown. We shall denote the two modes the $1+1+1$ and the $0+3$ configuration, respectively. In addition we find a third mode, where one soliton moves as a free entity and two solitons travels in a bunched configuration. We shall denote this mode a $1+2$ configuration. The velocity of the single soliton is different from that of the two-soliton bunch. Figure 12 shows the situation where the single soliton and the two-soliton

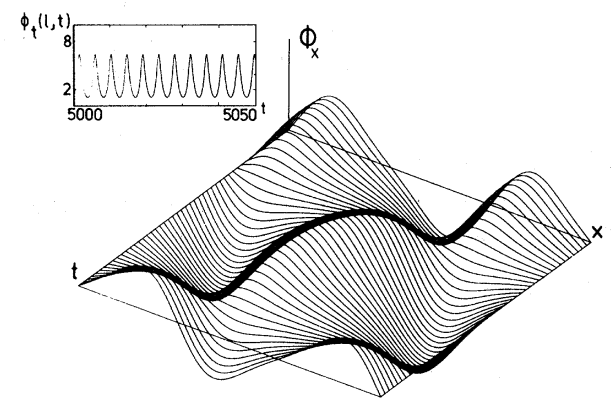

FIG. 10. Numerical solution of Eq. (2.10) with $\gamma=0.2, \alpha=0.05, \beta=0.02, \eta=0$, and $l=6$, showing the symmetric mode $(1+1+1)$ on the third ZFS in terms of $\phi_{x}(x, t)$ for 10 time units. The inset shows $\phi_{t}(l, t)$ for 50 time units.

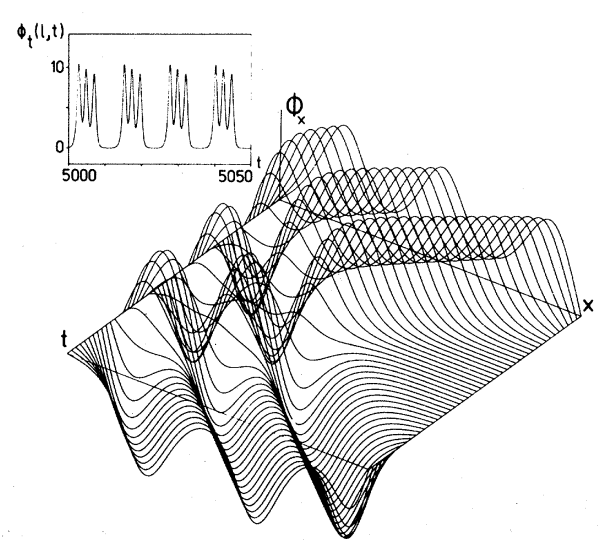

FIG. 11. Numerical solution of Eq. (2.10) with $\gamma=0.35, \alpha=0.05, \beta=0.02, \eta=0$, and $l=6$, showing the bunched mode $(0+3)$ on the third ZFS in terms of $\phi_{x}(x, t)$ for 10 time units. The inset shows $\phi_{t}(l, t)$ for 50 time units.

bunch have the maximum separation. At later times the picture looks much like that in Fig. 11. This $1+2$ configuration is only found stable in a narrow range of $\gamma$ values as seen in Fig. 13 where the first harmonic of the power versus $\gamma$ is shown. The $1+2$ configuration manifests itself as rapid oscillations in the first harmonic in the interval $0.34<\gamma<0.41$. Figure 13 is obtained in the same manner as Fig. 7. In Fig. 14 the first, second, and third harmonics are shown as functions of time for a number of selected $\gamma$ values in the range $0.34<\gamma<0.42$. For $\gamma=0.34$ [Fig. 14(a)] and $\gamma=0.42$ [Fig. 14(d)], the first harmonic settles to a constant value larger than the second and third harmonics. This implies the $0+3$ configuration for these $\gamma$ values. In Fig. 14(b) $(\gamma=0.36)$ and Fig.

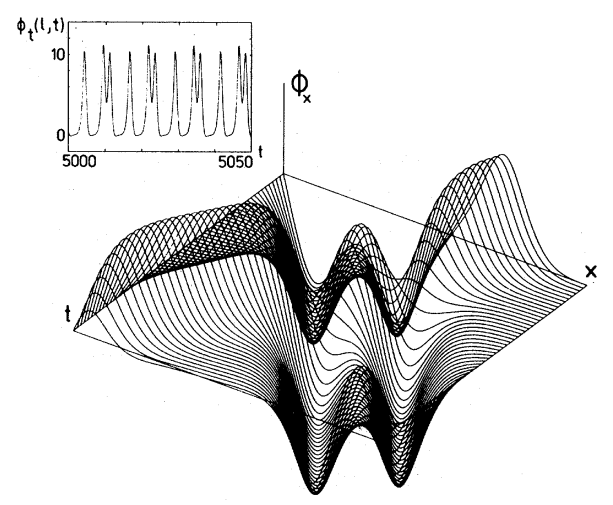

FIG. 12. Numerical solution of Eq. (2.10) with $\gamma=0.40, \beta=0.02, \eta=0$, and $l=6$, showing the $(1+2)$ bunched configuration on the third ZFS in terms of $\phi_{x}(x, t)$ for 10 time units. The inset shows $\phi_{t}(l, t)$ for 50 time units. 


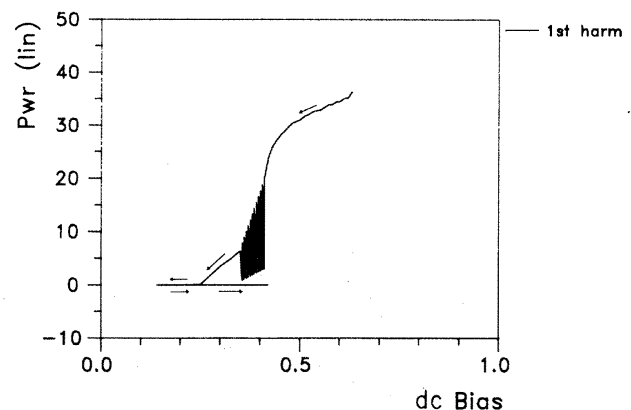

FIG. 13. First harmonic of the power vs the dc bias $\gamma$ on the third ZFS obtained by numerical solution of Eq. (2.10) with parameters for sample 1. The arrows indicate the direction in which the bias current was varied. The rapidly oscillating range indicates the coexistence of two adjacent frequencies in the signal.

14(c) $(\gamma=0.40)$, an oscillatory variation in the harmonics is found indicating the co-existence of oscillations at two adjacent frequencies. The twosoliton bunch moves with velocity $u$, thus given rise to the fundamental frequency $f_{1}=u / 2 l$. The single soliton moves with velocity $u+\Delta u$ giving rise to a second frequency separated from $f_{1}$ by $\Delta f=\Delta u / 2 l$. This simultaneous excitation of two adjacent frequencies was also found experimentally on both the third ZFS and the higher-order ZFS's. ${ }^{7,21}$ The experimentally observed separation was approximately $50 \mathrm{MHz}$. The oscillation period in Fig. 14(c) $(\gamma=0.40)$ indicates that $\Delta f \cong 23 \mathrm{MHz}$ in reasonable agreement with the experiments. [For this estimate Eq. (2.7) and $\omega_{0}$ (Table I) have been used.] For lower values of $\gamma$ the frequency separation $\Delta f$ gets smaller and vanishes completely at $\gamma=0.34$. When the bias current is decreased further, we find a transition to the symmetric mode at $\gamma=0.25$, in a manner similar to that found on the second ZFS. The symmetric mode is stable down to $\gamma=0.15$ where the solution switches from the third ZFS to the background curve. As on the second ZFS no spontaneous transition from the symmetric mode $(1+1+1)$ to a bunched mode $(0+3$ configuration) is found for increasing $\gamma$ values, nor does the $1+1+1$ mode enter the $2+1$ configuration when $\gamma$ varies through the interval $(0.34,0.42)$. This calculation was carried no further than to $\gamma=0.42$.

Introducing an external magnetic field $(\eta=0.1)$ did not change any quanlitative aspects of the transition from the $0+3$ configuration to the $1+2$ configuration. In Fig. 15 the time evolution of the harmonics is shown for two cases. In Fig. 15(a)
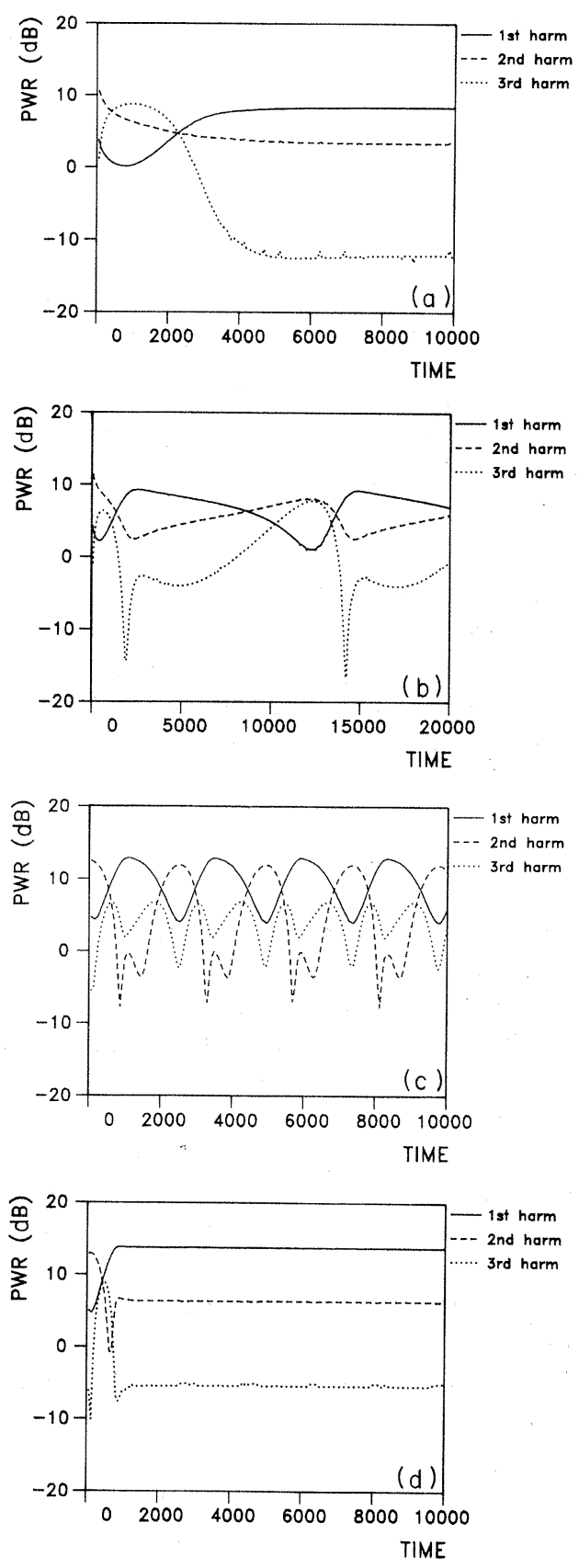

FIG. 14. Harmonics in power vs time on the third ZFS obtained by numerical solution of Eq. (2.10) with $\alpha=0.05, \beta=0.02, \eta=0, l=6$, and (a) $\gamma=0.34$, (b) $\gamma=0.36$, (c) $\gamma=0.40$, and (d) $\gamma=0.42$.

$(\gamma=0.34, \eta=0.1)$ steady state is reached earlier than for $\gamma=0.34$ and $\eta=0$ [Fig. 14(a)]. Also the magnitudes of the second and third harmonics are interchanged. The oscillatory behavior of the harmonics in Fig. 15(b) $(\gamma=0.40, \eta=0.1)$ is also some- 

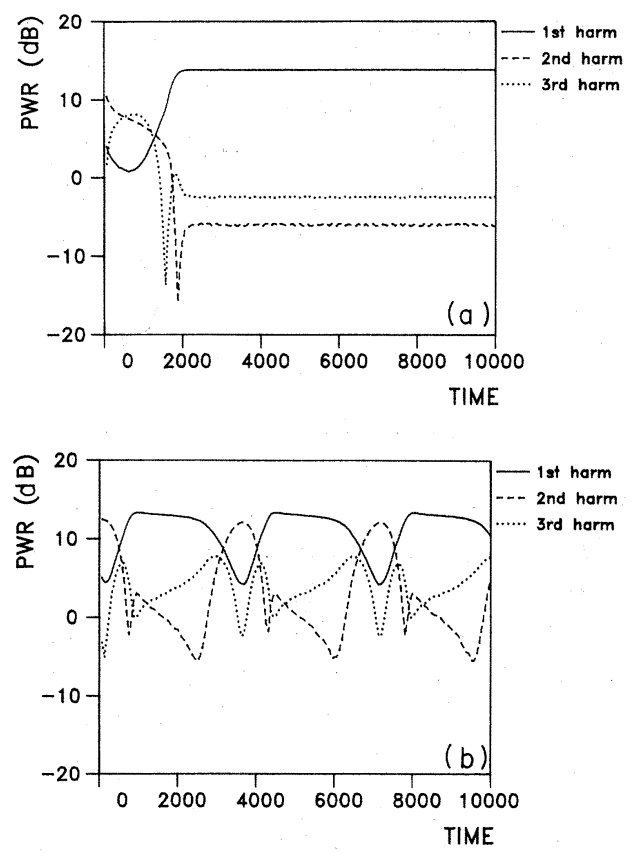

FIG. 15. Harmonics in power vs time on the third ZFS obtained by numerical solution of Eq. (2.10) with $\alpha=0.05, \beta=0.02, \eta=0.1, l=6$, and (a) $\gamma=0.34$ and (b) $\gamma=0.40$.

what modified compared to the behavior in Fig. 14(c) $(\gamma=0.40, \eta=0)$. We found the position and the range of stability of the $2+1$ mode unchanged as a small external magnetic field was applied. Also the range of stability shows no sensitive dependence on the initial conditions as long as these have the $0+3$ or the $2+1$ configuration.

\section{RESULTS FOR THE SHORTER JUNCTION $(l=2)$}

Experimental results are also available for a junction of relative short length $(l=2)$. The relevant parameters for this sample (no. 2) are given in Table I. We have performed numerical calculations similar to those described in Sec. III in order to ascertain whether the soliton picture applies for shorter junctions too. One might expect the soliton confinement to be more difficult on these junctions. ${ }^{22}$

In Fig. 16 we show the experimentally ${ }^{7}$ (solid curve) and numerically (points) obtained $I-V$ curve. Both in the experiments and in the computations only three ZFS's were found. There is a good agreement between the shape and position, whereas the height of the computed steps is generally lower

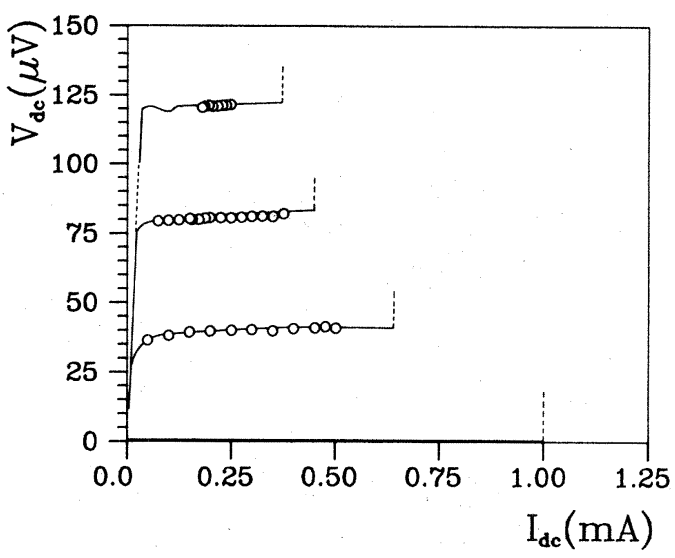

FIG. 16. dc voltage vs applied bias current for sample $2(l=2)$, showing the three ZFS's. Circles indicate the numerical results and the solid line represents the experimental results.

than the measured ones. Experimentally the short junction showed the same characteristic features as found on the longer junction-emission of microwave power with the same fundamental frequency on all ZFS's. Thus, we expect the bunched-soliton configurations to be stable also on the short junction. In Figs. 17-19 we show typical results of the oscillatory behavior on the three steps. Approximately half a period of steady-state oscillation on the first ZFS is shown in Fig. $17(\gamma=0.4)$. The bunched configuration on the second ZFS is shown in Fig. $18(\gamma=0.3)$. When $\gamma$ was decreased a transition to the symmetric mode was seen for $\gamma=0.15$. The irreversible behavior found for the longer junction is also seen here. Thus, no transition from symmetric mode to bunched mode is found for in-

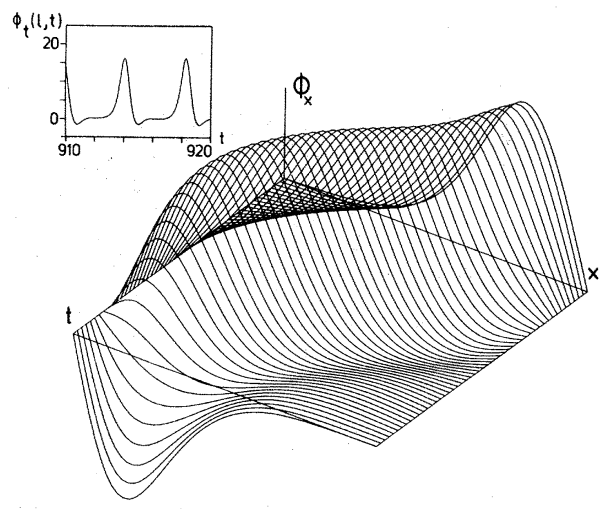

FIG. 17. Numerical solution of Eq. (2.10) with $\gamma=0.4, \alpha=0.012, \beta=0.01, \eta=0$, and $l=2$, showing approximately half a period of oscillation on the first ZFS plotted in terms of $\phi_{x}(x, t)$ for 2 time units. The inset shows $\phi_{t}(l, t)$ for 10 time units. 


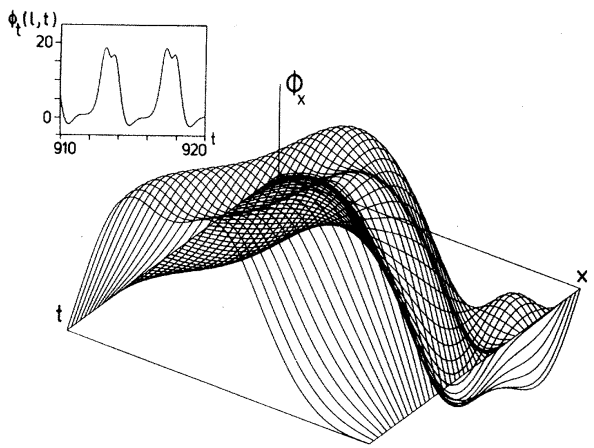

FIG. 18. Numerical solution of Eq. (2.10) with $\gamma=0.3, \alpha=0.012, \beta=0.01, \eta=0$, and $l=2$, showing the bunched mode on the second ZFS in terms of $\phi_{x}(x, t)$ for two time units. The inset shows $\phi_{t}(l, t)$ for 10 time units.

creasing $\gamma$. However, introduction of a small asymmetry in the model, through the boundary conditions, e.g., again removes the hysteresis. On the third ZFS only the bunched mode was found to be stable, shown in Fig. 19 for $\gamma=0.25$.

A comparison between the measured and calculated power emission at the fundamental frequency $f_{1}$ is shown in Fig. 20 versus the bias current. The calculated output power is determined through the relation $P=V^{2}\left(f_{1}\right) / 2 R_{L}$, where we use the load resistance $R_{L}$ as a fitting parameter. Using the value indicated by the arrow we find $R_{L}=17 \mathrm{k} \Omega$ which indicates that the open-circuit boundary condition [ $\eta=0$ in Eq. (2.16)] is a good approximation. This result also shows that the experimental matching to the ambient microwave circuit is

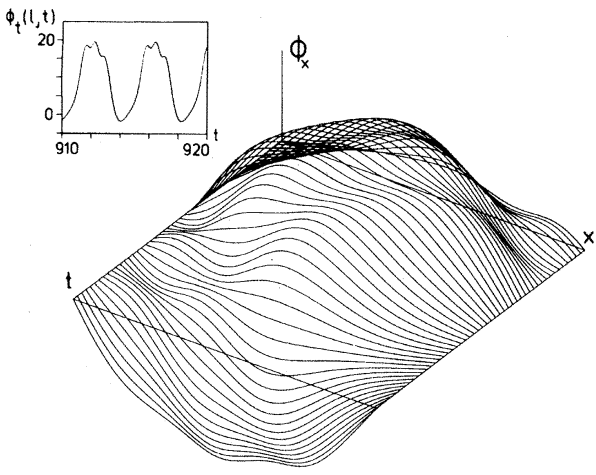

FIG. 19. Numerical solution of Eq. (2.10) with $\gamma=0.25, \alpha=0.012, \beta=0.01, \eta=0$, and $l=2$, showing the bunched mode on third ZFS in terms of $\phi_{x}(x, t)$ for three time units. The vertical scale is half of that of Figs. 17 and 18. The inset shows $\phi_{t}(l, t)$ for 10 time units. bad. In Fig. 20 the solid curves are the numerical results and the points indicate the experimental observations. The agreement is good for the first ZFS, but gets worse for the second and third ZFS. However, the qualitative behavior and the relative power levels are well reproduced.

\section{CONCLUDING REMARKS}

By detailed numerical calculations we have shown that the SJ model, which leads to a perturbed sine-Gordon equation, is able to explain recent experimental observations on junctions of different length. We conclude that the soliton picture applies well on both relatively long $(l=6)$ and intermediate-length $(l=2)$ junctions. Our calculations show the existence of the bunched-soliton configuration which is crucial for the understanding of the internal dynamics of the junctions. The bunched oscillation gives rise to a signal with fundamental frequency $f_{1}$, while the symmetric mode found on the lower part of the $N$ th ZFS yields the frequency $N f_{1}$. We remark that even though the symmetric modes shown in Figs. 5 and 10 are solutions of the perturbed sine-Gordon equation [Eq. (2.10)] it might be more natural to interpret them as current-driven cavity modes. ${ }^{23}$ Introduction of a small asymmetry via the boundary conditions, representing a weak magnetic field, e.g.,

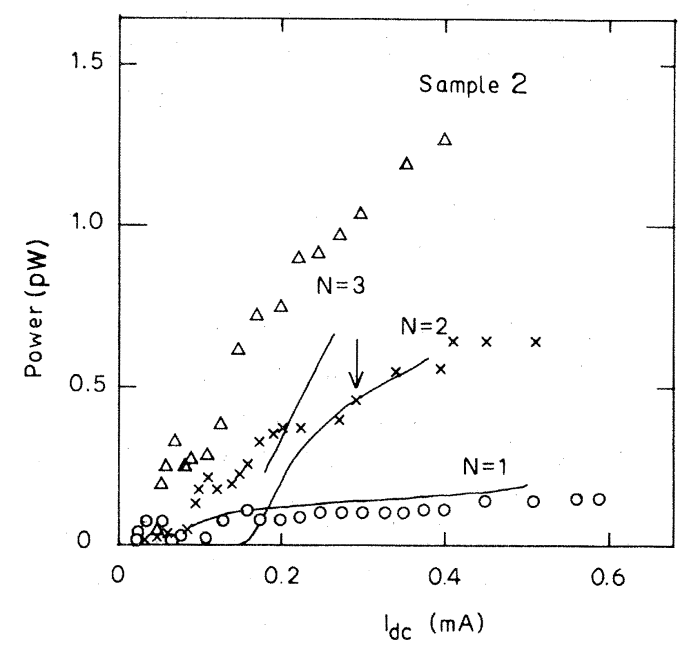

FIG. 20. Microwave power emitted from the short junction $(l=2)$ at the fundamental frequency $f_{1} . \quad N=1$, 2 , and 3 indicates the first, second, and third ZFS, respectively. The discrete points are experimental results and the solid lines are the numerical results. The arrow shows the power value used as a fitting parameter (see text). 
yields a junction response to variations in the applied bias current in agreement with experiments. Also the bunched-soliton configurations on the third ZFS explain the appearance of two simultaneous signals in the experiments. Finally we mention that some work regarding the detailed effects of the loading of power from the solitons to the ambient microwave circuit still remains, also it would be interesting to see if the recently proposed $^{20}$ soliton picture of the Fiske steps applies when the calculations are followed into steady state.

\section{ACKNOWLEDGMENTS}

It is our pleasure to thank B. Dueholm, O. Levring, J. Mygind, N.F. Pedersen, and M. Cirillo very much for many useful discussions and for giving permission to use their unpublished experimental results. Also we would like to express our deep gratitude to A.C. Scott and J.C. Eilbeck who participated in the initial phase of the present study and have shown a continued interest in our work. The work was supported in part by the Danish Natural Science Research Council.

\section{APPENDIX}

We have used an implicit finite-difference methods in order to solve Eq. (2.10) numerically. Denoting the restriction of $\phi(x, t)$ to a square mesh by $\phi_{i}^{n}=\phi(i h, n k)$, we get the following approximations for the derivatives:

$$
\begin{aligned}
& \phi_{t}=\frac{1}{2 k}\left(\phi_{i}^{n+1}-\phi_{i}^{n-1}\right)+O\left(k^{2}\right), \\
& \phi_{t t}=\frac{1}{k^{2}}\left(\phi_{i}^{n+1}-2 \phi_{i}^{n}+\phi_{i}^{n-1}\right)+O\left(k^{2}\right), \\
& \phi_{x x}=\frac{1}{2 h^{2}}\left(\phi_{i+1}^{n+1}-2 \phi_{i}^{n+1}+\phi_{i-1}^{n+1}+\phi_{i+1}^{n-1}-2 \phi_{i}^{n-1}+\phi_{i-1}^{n-1}\right)+O\left(h^{2}+k^{2}\right), \\
& \phi_{x x t}=\frac{1}{2 k h^{2}}\left(\phi_{i+1}^{n+1}-2 \phi_{i}^{n+1}+\phi_{i-1}^{n+1}-\phi_{i+1}^{n-1}+2 \phi_{i}^{n-1}-\phi_{i-1}^{n-1}\right)+O\left(h^{2}+k^{2}\right) .
\end{aligned}
$$

Substituting Eqs. (A1) - (A4) in Eq. (2.10) and negliecting $O\left(h^{2}\right)$ and $O\left(k^{2}\right)$, we get the following system of equations:

$$
\begin{gathered}
c_{1} \phi_{i+1}^{n+1}+c_{2} \phi_{i}^{n+1}+c_{1} \phi_{i-1}^{n+1}=c_{3}\left(\phi_{i+1}^{n-1}+\phi_{i-1}^{n-1}\right)-c_{4} \phi_{i}^{n-1}+c_{5} \phi_{i}^{n}+c_{6}\left(\sin \phi_{i}^{n}-\gamma\right), \\
i=1,2, \ldots, N, n=0,1, \ldots
\end{gathered}
$$

where

$$
\begin{aligned}
& c_{1}=\beta+k, \quad c_{2}=-\left(\alpha h^{2}+2 h^{2} / k+2 \beta+2 k\right), \quad c_{3}=\beta-k, \\
& c_{4}=\left(\alpha h^{2}-2 h^{2} / k+2 \beta-2 k\right), \quad c_{5}=-4 h^{2} / k, \quad c_{6}=2 h^{2} k .
\end{aligned}
$$

The boundary conditions are treated by introduction of imaginary points and Eq. (A5) is solved in a standard manner by means of the tridiagonal algorithm. ${ }^{24}$ The nonlinear term in Eq. (A5) is evaluated by a predictor-corrector loop. First, Eq. (A5) is used to compute the predictor $\widetilde{\phi}_{i}^{n+1}$, then this solution is reused in Eq. (A5), but now with the nonlinear term replaced by the average $\frac{1}{2}\left(\sin \widetilde{\phi}_{i}^{n+1}+\sin \phi_{i}^{n-1}\right)$. The accuracy of the numerical scheme has been checked by a systematic halving and doubling of time and space steps. In the results for the longer junction we have used 0.025 for the time step and 0.05 for the space step. For the shorter junction these values were 0.02 and 0.04 , respectively. 
*Present address: Center for Nonlinear Studies, Los Alamos National Laboratory, University of California, Los Alamos, New Mexico 87545.

†Present address: Danish Post \& Telegraph's Telecomminications Laboratory, Ågade 154, 2200 Copenhagen N, Denmark.

${ }^{1}$ T. A. Fulton and R. C. Dynes, Solid State Commun. 12, 57 (1973).

${ }^{2}$ T. A. Fulton and L. N. Dunkleberger, Rev. Phys. Appl. 9, 299 (1974).

${ }^{3}$ K. Nakajima, Y. Onodera, T. Nakamura, and R. Sato, J. Appl. Phys. 45, 4095 (1974).

${ }^{4} \mathrm{~K}$. Nakajima, T. Yamashita, and Y. Onodera, J. Appl. Phys. 45, 3141 (1974).

${ }^{5}$ S. N. Erné and R. D. Parmentier, in Proceedings of the 1980 Applied Superconductivity Conference, IEEE Trans. Mag. MAG-17, 804 (1981); J. Appl. Phys. 51, 5025 (1980); 르, 1091 (1981).

${ }^{6}$ M. Cirillo, R. D. Parmentier, and B. Savo, Physica D3, 565 (1981).

${ }^{7}$ B. Dueholm, O. A. Levring, J. Mygind, N. F. Pedersen, O. H. Soerensen, and M. Cirillo, Phys. Rev. Lett. 46, 1299 (1981).

${ }^{8}$ G. Costabile, A. M. Cucolo, S. Pace, R. D. Parmentier, B. Savo, and R. Vaglio, in SQUID-80, edited by H. D. Hahlbohm and H. Lubbig (de Gruyter, Berlin, 1980), p. 147; S. N. Erné, A. Ferrigno, T. F. Finnegan, and R. Vaglio, in Proceedings of the Sixteenth International Conference on Low Temperature Physics, Los Angeles, 1981 [Physica B + C, 108, 1301 (1981)].

${ }^{9}$ J. T. Chen, T. F. Finnegan, and D. N. Langenberg, in Proceedings of the International Conference on Superconductivity, Stanford, 1969, edited by F. Chilton (North-Holland, Amsterdam, 1971), p. 413; Physica $\underline{55}, 413$ (1971); J. T. Chen and D. N. Langenberg, in Proceedings of the Thirteenth International Conference on Low Temperature Physics, LT13, 1972, edited by K. D. Timmerhaus, W. J. Sullivan, and E. F. Hammel (Plenum, New York, 1974), Vol. 3, p. 289.

10D. D. Coon and M. D. Fiske, Phys. Rev. 138, A744 (1965).

${ }^{11}$ P. L. Christiansen, P. S. Lomdahl, A. C. Scott, O. H. Soerensen, and J. C. Eilbeck, Appl. Phys. Lett. $\underline{39}$, 108 (1981).

${ }^{12}$ C. S. Owen and D. J. Scalapino, Phys. Rev. 164, 538
(1967).

${ }^{13}$ B. D. Josephson, Adv. Phys. 14, 419 (1965).

${ }^{14}$ A. C. Scott, F. Y. F. Chu, and S. A. Reible, J. Appl. Phys. 47, 3272 (1976).

${ }^{15}$ T. A. Fulton, in Superconductor Applications: SQUIDs and Machines, edited by B. B. Schwartz and S. Foner (Plenum, New York, 1977), p. 125.

${ }^{16}$ A. C. Scott, Solid-State Electron. 7, 137 (1964).

${ }^{17}$ T. Yogi and J. E. Mercereau, IEEE Trans. Magn. MAG-17, 931 (1981); R. F. Broom and P. Wolf, Phys. Rev. B 16, 3100 (1977).

${ }^{18} \mathrm{An}$ initial condition which is not an exact solution of the perturbed sine-Gordon equation will in general break up into plasmons, breathers, and kinks (antikinks). Since plasmons and breathers have $\left\langle\phi_{t}\right\rangle=0$ over one period of oscillation, they cannot draw energy from the bias source and eventually they will be damped out. Choosing $\phi(x, t) \equiv \sin ^{-1} \gamma$ will reduce plasmon generation because it is the correct ground state for the perturbed system.

${ }^{19} \mathrm{This}$ value is not very important. It is chosen only to assure that the determination of the upper stability limit for oscillations is independent of the choice of initial conditions.

${ }^{20}$ The value $\eta=0.1$ used here corresponds to what is applied in the $H_{\mathrm{ex}} \cong 1.3 \mathrm{~A} / \mathrm{m}$. This is small compared to what is applied in the study of Fiske steps where $\eta$ is of the order 1. See, e.g., Ref. 7 and B. Dueholm, E. Joergensen, O. A. Levring, J. Mygind, N. F. Pedersen, M. R. Samuelsen, O. H. Olsen, and M. Cirillo, in Proceedings of the Sixteenth International Conference on Low Temperature Physics, Los Angeles, 1981 [Physica B + C 108, 1303 (1981)].

${ }^{21}$ P. S. Lomdahl, O. H. Soerensen, P. L. Christiansen, A. C. Scott, and J. C. Eilbeck, Phys. Rev. B 24, 7460 (1981).

${ }^{22}$ O. H. Soerensen, P. S. Lomdahl, and P. L. Christiansen, in Proceedings of Sixteenth International Conference on Low Temperature Physics, Los Angeles, 1981 [Physica B + C 108, 1299 (1981)].

23J. J. Chang and J. T. Chen, Phys. Rev. B 22, 2392 (1980).

${ }^{24}$ See, e.g., W. F. Ames, Numerical Methods for Partial Differential Equations (Academic, New York, 1977), p. 52 . 